\title{
LOW MUSCLE MASS IN OLDER MEN: THE ROLE OF LIFESTYLE, DIET AND CARDIOVASCULAR RISK FACTORS
}

\author{
J.L. ATKINS ${ }^{1}$, P.H. WHINCUP' ${ }^{2}$ R.W. MORRIS ${ }^{1}$, S.G. WANNAMETHEE ${ }^{1}$
}

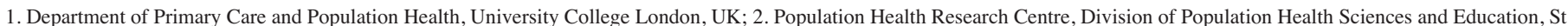

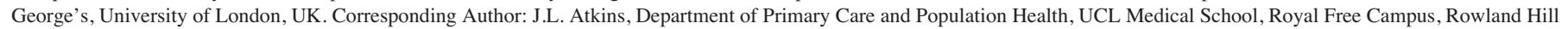
Street, London NW3 2PF, UK. Telephone: 02077940500 Ext 34389. Fax: 0207794 1224. E-mail: janice.atkins.11@ucl.ac.uk

\begin{abstract}
Objective: To explore associations between low muscle mass and a wide range of lifestyle, dietary and cardiovascular risk factors in older men including metabolic risk factors, markers of inflammation, endothelial dysfunction and coagulation. Design: Cross-sectional study. Setting: British Regional Heart Study. Participants: 4252 men aged 60-79 years. Measurements: Participants attended a physical examination in 1998-2000, and completed a general questionnaire and a food frequency questionnaire. Low muscle mass was assessed by two measures: midarm muscle circumference (MAMC) and fat-free mass index (FFMI). Associations between risk factors and low muscle mass were analysed using logistic regression. Results: Physical inactivity, insulin resistance, C-reactive protein, von Willebrand factor and fibrinogen were associated with significantly increased odds of low MAMC and FFMI after adjustment for body mass index, lifestyle characteristics and morbidity. Those with higher percent energy intake from carbohydrates showed decreased odds of low MAMC (OR: 0.73, 95\% CI: 0.55-0.96) and FFMI (OR: 0.76, 95\% CI: 0.58-0.99). Other dietary variables, smoking, alcohol intake, D-dimer, interleukin 6 and homocysteine showed no important associations with MAMC and FFMI. Conclusion: Increasing physical activity, consuming a diet with a high proportion of energy from carbohydrates, and taking steps to prevent insulin resistance and reduce inflammation and endothelial dysfunction may help to reduce the risk of low muscle mass in older men.
\end{abstract}

Key words: Muscle mass, lifestyle, diet, cardiovascular risk factors, aging.

\section{Introduction}

Sarcopenia, the age-associated loss of muscle mass and function, is associated with increased risks of mortality, functional impairment and physical disability (1-4) and accounts for substantial healthcare expenditure (5). Sarcopenia is common in older people with prevalence estimates from 5$13 \%$ among $60-70$ year olds, rising to $11-50 \%$ for those over 80 years (6). This variability in prevalence is related to a range of measurement methods and cut-points used to define sarcopenia (6), since an operational definition has not yet been adopted (7).

Sarcopenia has a multifactorial etiology which is not fully understood. Obesity is a strong determinant of sarcopenia and risk is increased by physical inactivity, current smoking, and low protein intake $(1,8,9)$. Additionally, low levels of energy intake, vitamin $\mathrm{D}$, antioxidants and omega-3 polyunsaturated fatty acids may be important in the development of sarcopenia (10). Recent evidence also suggests cardiovascular and inflammatory biomarkers are important contributors to sarcopenia progression (11). In addition, there is a suggestion from clinical studies that endothelial dysfunction may also contribute to sarcopenia (12) but less has been studied in the general population.

It is important to establish the contributions of a wide variety of potential low muscle mass determinants as well as potential mechanisms linking sarcopenia to mortality to inform the development of specific prevention and intervention strategies in older people. The aim of this study was to explore the associations between an extensive range of lifestyle and dietary risk factors associated with low muscle mass and to explore the role of novel cardiovascular risk markers (including metabolic risk factors, markers of inflammation, endothelial dysfunction and coagulation) in a population of older men. We used two separate assessments of muscle mass, based on arm measurements and bioelectrical impedance.

\section{Methods}

\section{Participants and study design}

The British Regional Heart Study is a prospective study investigating cardiovascular disease, in a socioeconomically and geographically representative sample of 7735 British men, from 24 towns in Great Britain (13). This cohort was first examined in 1978-80. Twenty years later, in 1998-2000, 4252 men (77\% of survivors), then aged $60-79$ years, attended a physical examination, provided a fasting blood sample, completed a general questionnaire and a food frequency questionnaire (14). Men provided written informed consent, in accordance with the Declaration of Helsinki. Ethical approval was obtained from relevant local research ethics committees.

\section{Anthropometric measurements}

Height, weight, waist circumference, fat-free mass, midupper arm circumference and triceps skinfold thickness were measured at re-examination in 1998-2000, as described previously (3). Two muscle mass markers were used: midarm muscle circumference (MAMC) and fat-free mass index (FFMI). MAMC $(\mathrm{cm})$ was calculated as: midupper arm circumference $(\mathrm{cm})-0.3142 \mathrm{x}$ triceps skinfold thickness $(\mathrm{mm})$ 
(15). Midupper arm circumference was measured once using an arm pendant. Triceps skinfold thickness was based on the average of two measurements. Fat-free mass was determined by bioelectrical impedance analysis (BIA) using a Bodystat 500 (Bodystat Ltd, Douglas, UK) and the Deurenberg et al equation (16). Fat-free mass was normalized for height by dividing by height $\left(\mathrm{m}^{2}\right)$ to give FFMI $\left(\mathrm{kg} / \mathrm{m}^{2}\right)(17)$. A standard statistical approach was used to define low muscle mass (participants below the first quartile of the distribution of MAMC or FFMI) since no consensus definition of sarcopenia has yet been adopted (7). However, MAMC correlates strongly with more accurate dual-energy X-ray absorptiometry measures of lean mass (18). In addition, the European Working Group on Sarcopenia has approved BIA measures as a portable alternative to dual-energy X-ray absorptiometry (7).

\section{Dietary assessment}

Dietary intake data were obtained using a food frequency questionnaire; development and validation have been described (19-21). Participants reported usual frequency of consumption of 86 food and drink types. Nutrient intakes were derived using a validated computer program to calculate total nutrient composition of foods consumed (22). In addition, participants were asked to indicate how often they consumed fresh fruit and vegetables - rarely/never, monthly, or 1, 2, 3, 4, 5, 6, or 7 days per week. Daily consumption was classified as 7 days per week. The multivariate nutrient density model was used to adjust macronutrients for energy intake; carbohydrates, fats and protein were expressed as percentages of energy (\% kcal) and energy intake was added to the logistic regression models for all dietary variables (23). Plasma vitamin $\mathrm{C}$ and $\mathrm{E}$ were also measured using high-performance liquid chromatography (22).

\section{Cardiovascular disease risk factors}

Cigarette smoking, physical activity, alcohol intake and social class were self-reported via questionnaire and blood lipids and blood pressure were measured at the physical examination as described elsewhere $(3,13,24)$. Men were classified into four smoking groups (never; long-term ex; recent ex; current). Current physical activity was classified in six groups based on exercise frequency and intensity (inactive; occasional; light; moderate; moderately vigorous; vigorous). Alcohol intake was classified into five groups based on the number and frequency of beverages consumed (none; occasional; light; moderate; heavy). Social class was split into three groups (manual; non-manual; armed forces) based on the longest held occupation using the Registrar General's occupational classification. Forced expiratory volume in 1 second $\left(\mathrm{FEV}_{1}\right)$ measured lung function and was heightstandardised to the mean height in this study $(1.72 \mathrm{~m})$.

The homeostasis model assessment was calculated to estimate insulin resistance (HOMA-IR) (25). Metabolic syndrome was classified according to the National Cholesterol Education Program definition (26); $\geq 3$ metabolic risk factors as follows: 1) Central obesity: waist circumference $>102 \mathrm{~cm}$; 2) Fasting plasma glucose $>6.1 \mathrm{mmol} / \mathrm{L}$; 3) Serum triglyceride $>1.7 \mathrm{mmol} / \mathrm{L}$; 4) Serum high density lipoprotein (HDL) cholesterol <1.04 mmol/L; and 5) Hypertension: blood pressure $>130 / 85 \mathrm{mmHg}$ or using blood pressure medication. Plasma concentrations of C-reactive protein (CRP), tissue plasminogen activator (t-PA) antigen, D-dimer, von Willebrand factor (vWF), fibrinogen, interleukin 6 (IL-6) and homocysteine were measured as described previously $(22,27,28)$.

\section{Morbidity}

Participant questionnaires provided self-reported data on doctor diagnosis of heart attack, angina, stroke, diabetes, heart failure and cancer. Prevalent cardiovascular disease (CVD) was defined as heart attack, angina or stroke diagnosis, and prevalent coronary heart disease (CHD) as heart attack or angina diagnosis. Participants were asked to rate their present state of health as excellent, good, fair or poor and if they had any long-standing illness or disability. Mobility limitation was determined by whether participants stated they had difficulty either going up/down stairs or walking for a quarter of a mile on a level surface (17).

\section{Statistical methods}

Of the 4252 men attending the physical examination, 4204 had MAMC data and 4114 had FFMI data for analysis. Descriptive characteristics of participants were presented by quartiles of the distribution of MAMC and FFMI. Distributions of CRP, D-dimer, HOMA-IR, homocysteine and IL-6 were highly skewed and log-transformed. Tests for trend were performed fitting MAMC/FFMI continuously using regression analyses, adjusting for age and body mass index (BMI) fitted as continuous variables. Additionally, we tested for an interaction between sarcopenia and obesity, each fitted as binary variables, with obesity defined using an established cut-point $\left(\geq 30 \mathrm{~kg} / \mathrm{m}^{2}\right)$ (29). However no evidence of interaction was found for any risk factors.

Multiple logistic regression was performed to obtain odds ratios for associations between risk factors and low muscle mass (the lowest quartile of MAMC/FFMI). Continuous dietary, metabolic and inflammatory risk factors were divided into quartiles and odds ratios for low muscle mass are presented for the highest versus the lowest quartile. Models were adjusted for potential confounders, associated with low muscle mass, in a sequential manor, including age (model 1), BMI and lifestyle characteristics (model 2) and morbidity variables (model 3). In addition, energy intake was included in the models for all dietary variables assessed by the food frequency questionnaire. Age, BMI, FEV 1 and energy intake were fitted as continuous variables. Smoking, physical activity, alcohol, social class, CVD, diabetes, cancer and poor/fair health were fitted as categorical variables. Statistical analyses were performed using Stata 12.0 (Stata Corp, College Station, TX). 


\section{LOW MUSCLE MASS IN OLDER MEN: THE ROLE OF LIFESTYLE, DIET AND CARDIOVASCULAR RISK FACTORS}

\section{Results}

The study sample had a mean age of 68.7 years (range: 6079), MAMC of $26.5 \mathrm{~cm}$ (SD: 2.3), FFMI of $17.3 \mathrm{~kg} / \mathrm{m}^{2}$ (SD: 2.2) and BMI of $26.9 \mathrm{~kg} / \mathrm{m}^{2}$ (SD: 3.7). MAMC and FFMI were moderately correlated $(\mathrm{r}=0.41, \mathrm{p}<0.001)$. Participant characteristics by MAMC quartiles are in Table 1. MAMC was inversely associated with age and positively associated with BMI. After adjustment for age and BMI, MAMC was inversely associated with smoking, heavy drinking, physical inactivity and manual working class. Energy, fiber, vitamin C, iron, daily fruit and vegetable consumption and plasma vitamin $\mathrm{E}$ showed significant positive linear trends with MAMC, but percent energy from fat showed a significant inverse linear trend.
HOMA-IR and high glucose were the only metabolic variables significantly associated with MAMC. Evidence of a significant inverse linear trend was found between MAMC and homocysteine, CRP, t-PA, D-dimer, vWF, fibrinogen and IL-6, after age and BMI adjustment.

Similar, less consistent, linear trends were observed between variables and FFMI quartiles, compared to MAMC quartiles (Table 2). Morbidity prevalence of participants by quartiles of MAMC and FFMI appear in Supplementary Table 1 and 2 respectively. MAMC showed a significant positive association with $\mathrm{FEV}_{1}$ and a significant inverse association with CVD, CHD, heart failure, poor/fair self-rated health, long-standing illness/disability and mobility limitation, after age and BMI adjustment $(\mathrm{p}<0.01)$. In comparison, FFMI showed similar

Table 1

Participant Characteristics by MAMC Quartiles

\begin{tabular}{|c|c|c|c|c|c|c|}
\hline & & МАM & uartiles $(\mathbf{c m})$ & & & \\
\hline & $\begin{array}{l}\leq 24.95 \\
n=1051\end{array}$ & $\begin{array}{c}24.95-26.45 \\
n=1051\end{array}$ & $\begin{array}{c}26.45-27.97 \\
n=1051\end{array}$ & $\begin{array}{l}\geq 27.97 \\
n=1051\end{array}$ & $\begin{array}{l}\text { p-value for trend } \\
\text { (age adjusted) }\end{array}$ & $\begin{array}{c}\text { p-value for trend } \\
\text { (age \& BMI adjusted) }\end{array}$ \\
\hline Socio-demographic/Lifestyle varia & ables & & & & & \\
\hline Age (years) & $70.2 \pm 5.7$ & $69.3 \pm 5.5$ & $68.4 \pm 5.3$ & $67.0 \pm 5.0$ & $<0.001^{\mathrm{a}}$ & $<0.001^{\mathrm{b}}$ \\
\hline Obesity $\left(\mathrm{BMI} \geq 30 \mathrm{~kg} / \mathrm{m}^{2}\right)(\%)$ & 2.9 & 7.7 & 17.1 & 39.4 & $<0.001$ & $\mathrm{n} / \mathrm{a}$ \\
\hline Current smokers $(\%)$ & 17.9 & 14.1 & 9.8 & 9.4 & $<0.001$ & $<0.001$ \\
\hline Heavy drinkers $(\%)$ & 3.8 & 3.0 & 2.5 & 2.4 & 0.29 & 0.03 \\
\hline Physically inactive (\%) & 15.1 & 9.4 & 10.2 & 10.8 & 0.09 & $<0.001$ \\
\hline Manual workers (\%) & 53.2 & 49.1 & 50.1 & 50.8 & 0.30 & $<0.001$ \\
\hline Dietary variables & & & & & & \\
\hline Energy intake (kcal) & $2125.7 \pm 524.4$ & $2125.6 \pm 528.6$ & $2100.4 \pm 524.3$ & $2103.0 \pm 542.4$ & 0.41 & 0.02 \\
\hline Carbohydrates (\% kcal) & $53.1 \pm 6.9$ & $52.8 \pm 6.9$ & $52.5 \pm 6.9$ & $52.1 \pm 7.1$ & $<0.001$ & 0.06 \\
\hline Protein $(\% \mathrm{kcal})$ & $15.4 \pm 2.3$ & $15.7 \pm 2.4$ & $15.8 \pm 2.3$ & $15.8 \pm 2.3$ & 0.001 & 0.10 \\
\hline Fat $(\% \mathrm{kcal})$ & $30.5 \pm 6.1$ & $30.1 \pm 6.2$ & $29.9 \pm 6.3$ & $30.0 \pm 6.3$ & 0.99 & 0.04 \\
\hline Fiber (g/day) & $25.6 \pm 9.0$ & $26.1 \pm 8.6$ & $25.8 \pm 8.5$ & $26.2 \pm 8.9$ & 0.25 & $<0.001$ \\
\hline Vitamin C (mg/day) & $79.2 \pm 36.3$ & $82.8 \pm 36.3$ & $83.7 \pm 36.2$ & $87.1 \pm 39.5$ & $<0.001$ & 0.001 \\
\hline Vitamin E (mg/day) & $8.4 \pm 4.8$ & $8.4 \pm 4.8$ & $8.5 \pm 4.7$ & $8.5 \pm 5.0$ & 0.31 & 0.08 \\
\hline Iron (mg/day) & $14.0 \pm 5.1$ & $14.4 \pm 5.5$ & $14.1 \pm 5.0$ & $14.2 \pm 5.3$ & 0.26 & $<0.001$ \\
\hline Daily fruit intake (\%) & 40.5 & 42.8 & 44.7 & 44.2 & 0.002 & 0.01 \\
\hline Daily vegetable intake (\%) & 30.9 & 32.9 & 33.2 & 35.1 & 0.002 & $<0.001$ \\
\hline Plasma vitamin $\mathrm{C}(\mu \mathrm{mol} / \mathrm{L})$ & $30.1 \pm 28.9$ & $30.1 \pm 28.6$ & $31.0 \pm 27.9$ & $29.5 \pm 23.5$ & 0.39 & 0.26 \\
\hline Plasma vitamin $\mathrm{E}(\mu \mathrm{mol} / \mathrm{L})$ & $32.1 \pm 11.4$ & $33.8 \pm 12.0$ & $34.3 \pm 11.8$ & $33.8 \pm 12.2$ & 0.001 & 0.01 \\
\hline Metabolic variables & & & & & & \\
\hline HOMA-IR $^{\mathrm{c}}$ & $1.9(1.1-2.7)$ & $2.1(1.4-3.0)$ & $2.3(1.5-3.2)$ & $2.7(1.7-4.0)$ & $<0.001$ & 0.002 \\
\hline Central obesity (\%) & 9.0 & 19.5 & 31.9 & 54.7 & $<0.001$ & $\mathrm{n} / \mathrm{a}$ \\
\hline Hypertension (\%) & 53.8 & 56.1 & 62.9 & 63.4 & $<0.001$ & 0.32 \\
\hline High triglycerides $(\%)$ & 33.4 & 42.4 & 46.8 & 56.9 & $<0.001$ & 0.08 \\
\hline Low HDL (\%) & 15.2 & 18.7 & 21.3 & 27.9 & $<0.001$ & 0.77 \\
\hline High glucose $(\%)$ & 25.0 & 24.2 & 23.5 & 27.3 & 0.001 & 0.006 \\
\hline Metabolic Syndrome (\%) & 13.5 & 20.8 & 29.7 & 42.9 & $<0.001$ & 0.57 \\
\hline Inflammatory/biological markers & & & & & & \\
\hline $\mathrm{CRP}(\mathrm{mg} / \mathrm{L})^{\mathrm{c}}$ & $1.8(0.8-3.8)$ & $1.7(0.8-3.5)$ & $1.7(0.8-3.3)$ & $1.7(0.9-3.4)$ & 0.09 & $<0.001$ \\
\hline $\mathrm{t}-\mathrm{PA}(\mathrm{ng} / \mathrm{mL})$ & $10.5 \pm 4.6$ & $10.9 \pm 4.4$ & $11.2 \pm 4.5$ & $11.6 \pm 4.2$ & $<0.001$ & 0.01 \\
\hline D-dimer $(\mathrm{ng} / \mathrm{mL})^{\mathrm{c}}$ & $94.2(52.0-145.0)$ & $88.4(50.0-134.0)$ & $83.5(47.0-128.0)$ & $72.8(46.0-105.0)$ & 0.002 & $<0.001$ \\
\hline vWF (IU/dL) & $147.3 \pm 48.6$ & $141.2 \pm 46.1$ & $136.6 \pm 44.1$ & $134.0 \pm 44.4$ & $<0.001$ & $<0.001$ \\
\hline Fibrinogen $(\mathrm{g} / \mathrm{L})$ & $3.4 \pm 0.8$ & $3.3 \pm 0.8$ & $3.3 \pm 0.7$ & $3.2 \pm 0.7$ & $<0.001$ & $<0.001$ \\
\hline IL-6 $(\mathrm{pg} / \mathrm{mL})^{\mathrm{c}}$ & $2.6(1.6-3.8)$ & $2.5(1.6-3.4)$ & $2.6(1.5-3.4)$ & $2.4(1.6-3.3)$ & 0.53 & $<0.001$ \\
\hline Homocysteine $(\mu \mathrm{mol} / \mathrm{L})^{\mathrm{c}}$ & $13.2(10.4-16.0)$ & $12.7(10.3-14.9)$ & $12.6(10.3-14.7)$ & $12.2(10.0-14.2)$ & 0.002 & $<0.001$ \\
\hline
\end{tabular}

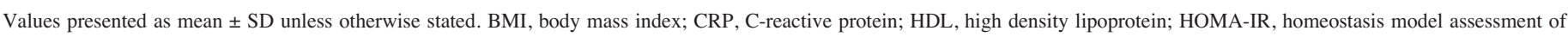

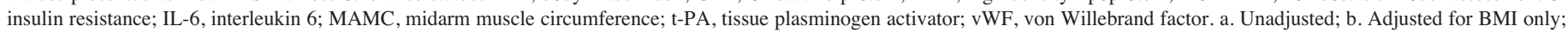
c. Log-transformed-geometric mean (interquartile range) presented. 
Table 2

Participant Characteristics by FFMI Quartiles

\begin{tabular}{|c|c|c|c|c|c|c|}
\hline & & FFMI Qus & les $\left(\mathrm{kg} / \mathbf{m}^{2}\right)$ & & & \\
\hline & $\begin{array}{l}\leq 15.96 \\
\mathrm{n}=1029 \\
\end{array}$ & $\begin{array}{c}15.96-17.09 \\
\mathrm{n}=1028\end{array}$ & $\begin{array}{c}17.09-18.36 \\
n=1029\end{array}$ & $\begin{array}{l}\geq 18.36 \\
n=1028 \\
\end{array}$ & $\begin{array}{l}\text { p-value for trend } \\
\text { (age adjusted) }\end{array}$ & $\begin{array}{c}\text { p-value for trend } \\
\text { (age \& BMI adjusted) }\end{array}$ \\
\hline Socio-demographic/Lifestyle varia & ables & & & & & \\
\hline Age (years) & $69.0 \pm 5.5$ & $68.8 \pm 5.5$ & $68.3 \pm 5.5$ & $68.7 \pm 5.5$ & $0.19^{\mathrm{a}}$ & $0.07^{\mathrm{b}}$ \\
\hline Obesity $\left(\mathrm{BMI} \geq 30 \mathrm{~kg} / \mathrm{m}^{2}\right)(\%)$ & 2.7 & 9.1 & 16.4 & 42.0 & $<0.001$ & $\mathrm{n} / \mathrm{a}$ \\
\hline Current smokers $(\%)$ & 16.4 & 12.8 & 11.4 & 10.2 & $<0.001$ & 0.12 \\
\hline Heavy drinkers (\%) & 3.0 & 3.2 & 2.8 & 3.3 & 0.77 & 0.52 \\
\hline Physically inactive (\%) & 13.4 & 10.8 & 9.1 & 12.6 & 0.28 & $<0.001$ \\
\hline Manual workers (\%) & 51.6 & 49.4 & 51.7 & 52.2 & 0.37 & $<0.001$ \\
\hline Dietary variables & & & & & & \\
\hline Energy intake (kcal) & $2140.0 \pm 523.6$ & $2108.4 \pm 514.4$ & $2107.6 \pm 529.7$ & $2097.5 \pm 552.2$ & 0.24 & 0.20 \\
\hline Carbohydrates (\% kcal) & $52.9 \pm 6.9$ & $52.7 \pm 7.0$ & $52.7 \pm 7.0$ & $51.9 \pm 6.8$ & $<0.001$ & 0.13 \\
\hline Protein $(\% \mathrm{kcal})$ & $15.4 \pm 2.4$ & $15.6 \pm 2.2$ & $15.7 \pm 2.3$ & $16.0 \pm 2.3$ & $<0.001$ & 0.02 \\
\hline Fat $(\% \mathrm{kcal})$ & $30.4 \pm 6.2$ & $30.0 \pm 6.0$ & $29.9 \pm 6.4$ & $30.3 \pm 6.1$ & 0.41 & 0.27 \\
\hline Fiber (g/day) & $25.6 \pm 8.9$ & $25.8 \pm 8.5$ & $26.1 \pm 8.6$ & $26.3 \pm 9.1$ & 0.08 & $<0.001$ \\
\hline Vitamin C (mg/day) & $80.5 \pm 37.5$ & $82.1 \pm 35.7$ & $83.3 \pm 36.2$ & $86.3 \pm 39.3$ & 0.002 & 0.21 \\
\hline Vitamin E (mg/day) & $8.6 \pm 5.0$ & $8.4 \pm 4.7$ & $8.3 \pm 4.7$ & $8.6 \pm 4.9$ & 0.05 & 0.03 \\
\hline Iron (mg/day) & $14.1 \pm 5.3$ & $14.2 \pm 5.1$ & $14.1 \pm 5.3$ & $14.3 \pm 5.1$ & 0.49 & 0.01 \\
\hline Daily fruit intake (\%) & 39.5 & 44.2 & 41.7 & 47.3 & $<0.001$ & 0.001 \\
\hline Daily vegetable intake (\%) & 30.0 & 33.9 & 32.7 & 35.0 & 0.02 & 0.002 \\
\hline Plasma vitamin $\mathrm{C}(\mu \mathrm{mol} / \mathrm{L})$ & $28.7 \pm 24.8$ & $31.2 \pm 30.4$ & $31.0 \pm 27.9$ & $29.4 \pm 22.6$ & 0.59 & 0.02 \\
\hline Plasma vitamin E $(\mu \mathrm{mol} / \mathrm{L})$ & $32.6 \pm 11.5$ & $33.8 \pm 12.2$ & $33.6 \pm 12.2$ & $34.3 \pm 11.8$ & 0.002 & 0.03 \\
\hline Metabolic variables & & & & & & \\
\hline HOMA-IR $^{c}$ & $2.0(1.2-2.9)$ & $2.2(1.4-3.0)$ & $2.3(1.4-3.2)$ & $2.7(1.6-4.0)$ & $<0.001$ & $<0.001$ \\
\hline Central obesity (\%) & 14.6 & 23.1 & 31.5 & 49.6 & $<0.001$ & $\mathrm{n} / \mathrm{a}$ \\
\hline Hypertension (\%) & 54.4 & 60.5 & 58.4 & 64.3 & 0.001 & 0.02 \\
\hline High triglycerides (\%) & 40.0 & 44.3 & 46.5 & 50.5 & $<0.001$ & $<0.001$ \\
\hline Low HDL (\%) & 15.5 & 17.6 & 22.8 & 27.7 & $<0.001$ & 0.47 \\
\hline High glucose $(\%)$ & 23.7 & 25.6 & 23.0 & 29.2 & 0.004 & 0.04 \\
\hline Metabolic Syndrome (\%) & 17.5 & 23.8 & 28.6 & 39.7 & $<0.001$ & $<0.001$ \\
\hline Inflammatory/biological markers & & & & & & \\
\hline $\mathrm{CRP}(\mathrm{mg} / \mathrm{L})^{\mathrm{c}}$ & $1.8(0.8-3.8)$ & $1.8(0.8-3.6)$ & $1.7(0.8-3.3)$ & $1.7(0.9-3.3)$ & 0.77 & $<0.001$ \\
\hline $\mathrm{t}-\mathrm{PA}(\mathrm{ng} / \mathrm{mL})$ & $11.2 \pm 4.7$ & $11.1 \pm 4.4$ & $10.7 \pm 4.3$ & $11.5 \pm 4.4$ & 0.01 & $<0.001$ \\
\hline D-dimer $(\mathrm{ng} / \mathrm{mL})^{\mathrm{c}}$ & $85.1(49.0-133.0)$ & $83.1(49.0-123.0)$ & $83.6(47.0-133.5)$ & $83.9(50.0-127.0)$ & 0.66 & 0.71 \\
\hline $\mathrm{vWF}(\mathrm{IU} / \mathrm{dL})$ & $144.6 \pm 48.2$ & $140.0 \pm 45.7$ & $136.6 \pm 43.2$ & $138.6 \pm 47.1$ & 0.230 & $<0.001$ \\
\hline Fibrinogen $(\mathrm{g} / \mathrm{L})$ & $3.4 \pm 0.8$ & $3.3 \pm 0.8$ & $3.2 \pm 0.7$ & $3.2 \pm 0.7$ & $<0.001$ & $<0.001$ \\
\hline $\operatorname{IL}-6(\mathrm{pg} / \mathrm{mL})^{\mathrm{c}}$ & $2.4(1.6-3.5)$ & $2.4(1.6-3.4)$ & $2.4(1.6-3.4)$ & $2.5(1.6-3.7)$ & 0.05 & $<0.001$ \\
\hline Homocysteine $(\mu \mathrm{mol} / \mathrm{L})^{c}$ & $12.9(10.2-15.5)$ & $12.5(10.1-14.7)$ & $12.5(10.1-14.3)$ & $12.8(10.4-14.8)$ & 0.50 & 0.11 \\
\hline
\end{tabular}

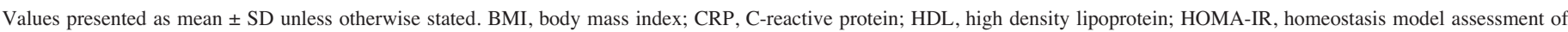

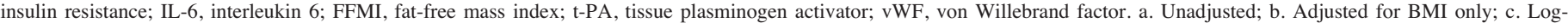
transformed values-geometric mean (interquartile range) presented.

patterns of association.

Multiple logistic regression analyses of risk factors and low MAMC or FFMI appear in Table 3. In the fully adjusted model, physical inactivity and manual work (but not current smoking or heavy drinking) were significantly associated with the odds of low MAMC. Men in the highest quartile (compared to the lowest) of percent energy from protein, vitamin C, plasma vitamin $\mathrm{E}$, and daily fruit consumers, were at significantly decreased odds of low MAMC, adjusting for age. However, after adjusting for BMI, lifestyle and morbidity variables, these associations were markedly attenuated and became nonsignificant. Men with high energy intake and high percentage energy from carbohydrates showed lower odds of low MAMC even after adjustment. By contrast, those with high percentage energy from fat showed higher odds of low MAMC. High
HOMA-IR and blood glucose were associated with increased odds of low MAMC after full adjustment. No independent association was seen with other metabolic risk factors. HOMAIR was strongly correlated with obesity $(r=0.45, p<0.001)$, which explains why the direction of association changed after BMI adjustment. High levels of CRP, vWF and fibrinogen were associated with increased odds of low MAMC after adjustment for BMI, lifestyle and morbidity variables. D-dimer, t-PA, IL-6 and homocysteine were not significantly associated with low MAMC.

In comparison to MAMC analyses, consistent significant associations were also observed between being in the lowest FFMI quartile and physical inactivity, percent energy from carbohydrates, HOMA-IR, CRP, vWF and fibrinogen in the final model (Table 3). In addition, plasma vitamin $C$, central 
Table 3

Multiple Logistic Regression Analysis of Factors Associated with Low MAMC and FFMI

\begin{tabular}{|c|c|c|c|c|}
\hline & \multicolumn{3}{|c|}{ Low MAMC (OR, 95\% CI) } & \multirow{2}{*}{$\begin{array}{c}\text { Low FFMI (OR, 95\% CI) } \\
\text { Model } 3 \\
\end{array}$} \\
\hline & Model 1 & Model 2 & Model 3 & \\
\hline \multicolumn{5}{|c|}{ Socio-demographic/Lifestyle variables } \\
\hline Current smokers & $1.87(1.54-2.28) * *$ & $1.12(0.87-1.44)$ & $1.01(0.78-1.32)$ & $0.94(0.73-1.22)$ \\
\hline Heavy drinkers & $1.55(1.04-2.30)^{*}$ & $1.52(0.93-2.49)$ & $1.54(0.92-2.56)$ & $0.80(0.47-1.36)$ \\
\hline Physically inactive & $1.43(1.15-1.76)^{* *}$ & $1.97(1.50-2.59)^{* *}$ & $1.69(1.26-2.27)^{* *}$ & $1.43(1.07-1.90)^{*}$ \\
\hline Manual workers & $1.14(0.99-1.31)$ & $1.31(1.10-1.56)^{* *}$ & $1.31(1.09-1.57)^{* *}$ & $1.04(0.87-1.24)$ \\
\hline \multicolumn{5}{|l|}{ Dietary variables } \\
\hline Energy intake ${ }^{\mathrm{a}}$ & $0.99(0.80-1.22)$ & $0.74(0.57-0.95)^{*}$ & $0.73(0.56-0.95)^{*}$ & $1.14(0.89-1.46)$ \\
\hline Carbohydrates $(\% \mathrm{kcal})^{\mathrm{ab}}$ & $1.17(0.95-1.43)$ & $0.72(0.56-0.94)^{*}$ & $0.73(0.55-0.96)^{*}$ & $0.76(0.58-0.99)^{*}$ \\
\hline Protein $(\% \mathrm{kcal})^{\mathrm{ab}}$ & $0.78(0.62-0.96)^{*}$ & $1.21(0.93-1.59)$ & $1.16(0.88-1.54)$ & $0.89(0.68-1.16)$ \\
\hline Fat $(\% \mathrm{kcal})^{\mathrm{ab}}$ & $1.18(0.95-1.46)$ & $1.33(1.03-1.72)^{*}$ & $1.31(1.00-1.71)^{*}$ & $1.24(0.94-1.60)$ \\
\hline Fiber $^{\text {ab }}$ & $0.81(0.65-1.00)$ & $0.93(0.70-1.22)$ & $0.97(0.73-1.30)$ & $0.85(0.64-1.12)$ \\
\hline Vitamin $C^{\mathrm{ab}}$ & $0.65(0.53-0.80)^{* *}$ & $0.94(0.73-1.22)$ & $0.92(0.70-1.21)$ & $0.98(0.76-1.28)$ \\
\hline Vitamin $E^{\mathrm{ab}}$ & $0.86(0.69-1.05)$ & $0.95(0.73-1.25)$ & $0.91(0.69-1.21)$ & $1.05(0.80-1.37)$ \\
\hline Iron $^{\text {ab }}$ & $0.84(0.65-1.08)$ & $1.03(0.75-1.42)$ & $1.01(0.72-1.41)$ & $0.77(0.56-1.06)$ \\
\hline Daily fruit intake ${ }^{\mathrm{b}}$ & $0.85(0.73-0.99)^{*}$ & $1.01(0.84-1.21)$ & $0.98(0.81-1.18)$ & $0.89(0.75-1.07)$ \\
\hline Daily vegetable intake ${ }^{\mathrm{b}}$ & $0.86(0.74-1.01)$ & $0.94(0.78-1.13)$ & $0.94(0.78-1.14)$ & $0.83(0.69-1.00)$ \\
\hline Plasma vitamin $\mathrm{C}^{\mathrm{a}}$ & $0.84(0.69-1.04)$ & $0.77(0.60-1.00)^{*}$ & $0.82(0.63-1.07)$ & $0.71(0.55-0.92)^{*}$ \\
\hline Plasma vitamin $\mathrm{E}^{\mathrm{a}}$ & $0.71(0.57-0.88)^{* *}$ & $0.87(0.67-1.13)$ & $0.88(0.67-1.15)$ & $0.93(0.72-1.20)$ \\
\hline \multicolumn{5}{|l|}{ Metabolic variables } \\
\hline HOMA-IR ${ }^{\mathrm{a}}$ & $0.37(0.30-0.46)^{* *}$ & $1.64(1.25-2.17)^{* *}$ & $1.60(1.19-2.17)^{* *}$ & $2.22(1.65-2.98)^{* *}$ \\
\hline Central obesity & $0.18(0.14-0.22)^{* *}$ & $1.07(0.79-1.44)$ & $0.95(0.70-1.30)$ & $2.01(1.52-2.67)^{* *}$ \\
\hline Hypertension & $0.72(0.63-0.84)^{* *}$ & $0.96(0.81-1.15)$ & $0.94(0.78-1.12)$ & $1.00(0.84-1.19)$ \\
\hline High triglycerides & $0.54(0.47-0.63) \div$ & $0.93(0.77-1.11)$ & $0.86(0.71-1.04)$ & $1.32(1.10-1.59)^{* *}$ \\
\hline Low HDL & $0.62(0.51-0.75)^{* *}$ & $1.02(0.81-1.30)$ & $1.00(0.78-1.28)$ & $0.98(0.77-1.24)$ \\
\hline High glucose & $0.96(0.81-1.13)$ & $1.37(1.11-1.68)^{* *}$ & $1.34(1.07-1.69)^{*}$ & $1.20(0.96-1.49)$ \\
\hline Metabolic Syndrome & $0.34(0.28-0.42) \ddagger$ & $0.99(0.77-1.27)$ & $0.89(0.69-1.16)$ & $1.30(1.02-1.66)^{*}$ \\
\hline \multicolumn{5}{|c|}{ Inflammatory/biological markers } \\
\hline $\mathrm{CRP}^{\mathrm{a}}$ & $0.89(0.73-1.10)$ & $1.66(1.28-2.15)^{* *}$ & $1.44(1.09-1.89)^{*}$ & $1.94(1.48-2.54)^{* *}$ \\
\hline $\mathrm{t}-\mathrm{PA}^{\mathrm{a}}$ & $0.46(0.40-0.61)^{* *}$ & $1.11(0.85-1.45)$ & $1.01(0.76-1.33)$ & $2.33(1.78-3.04)^{* *}$ \\
\hline D-dimer ${ }^{\mathrm{a}}$ & $1.15(0.93-1.43)$ & $1.14(0.88-1.48)$ & $1.03(0.79-1.36)$ & $0.91(0.70-1.19)$ \\
\hline $\mathrm{vWF}^{\mathrm{a}}$ & $1.49(1.21-1.83)^{* *}$ & $1.74(1.36-2.23)^{* *}$ & $1.67(1.29-2.17)^{* *}$ & $1.57(1.22-2.02)^{* *}$ \\
\hline Fibrinogen $^{a}$ & $1.52(1.24-1.87)^{* *}$ & $1.63(1.26-2.11)^{* *}$ & $1.48(1.13-1.95)^{* *}$ & $1.52(1.17-1.96)^{* *}$ \\
\hline IL-6 $6^{\mathrm{a}}$ & $1.10(0.90-1.36)$ & $1.33(1.02-1.73)^{*}$ & $1.23(0.94-1.63)$ & $1.13(0.86-1.47)$ \\
\hline Homocysteine $^{\mathrm{a}}$ & $1.23(1.00-1.51)$ & $1.22(0.95-1.58)$ & $1.20(0.92-1.57)$ & $1.09(0.84-1.41)$ \\
\hline
\end{tabular}

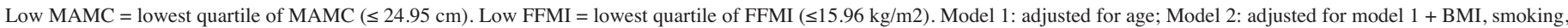

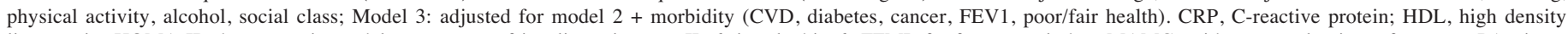

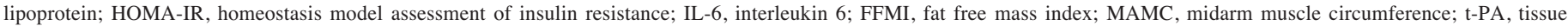
plasminogen activator; vWF, von Willebrand factor; $* \mathrm{p}<0.05 * * \mathrm{p}<0.01$; a. Highest vs. lowest quartile; b.Additionally adjusted for energy intake in all models.

obesity, high triglycerides, t-PA and the metabolic syndrome were significantly associated with low FFMI.

\section{Discussion}

We explored associations between clinically available and easily measureable markers of low muscle mass and a range of lifestyle, dietary and cardiovascular risk factors in older men. Based on consistent associations with low MAMC and FFMI, physical inactivity, low percent energy from carbohydrates, insulin resistance, inflammatory markers (CRP, fibrinogen) and endothelial dysfunction (vWF) are associated with low muscle mass, independent of age, obesity, lifestyle and morbidity variables.
Physical inactivity was the strongest lifestyle factor associated with low MAMC and FFMI, consistent with previous literature $(1,8,30)$. This highlights the need for interventions to increase activity in older adults. Current smoking was not significantly associated with low muscle mass. This conflicts with earlier studies $(1,30)$ which may not have fully adjusted for confounding variables, as in our study the effect of smoking on muscle mass disappeared after BMI adjustment. As found previously, heavy alcohol intake was not a risk factor for low muscle mass $(1,30)$.

A high percentage energy intake from carbohydrates was associated with decreased odds of low MAMC and FFMI. To our knowledge, this is the first suggestion that high percentage energy intake of this macronutrient may be protective against 


\section{JNHA: NUTRITION}

low muscle mass. Our findings are in keeping with the World Health Organization dietary recommendations of a low-fat high-carbohydrate diet, with $55-75 \%$ energy from carbohydrates (31). Consistent with literature suggesting energy intake is important for the prevention of low muscle mass (10) we found an association between low energy intake and low MAMC, emphasising the need for an adequate calorie intake in older adults. No other dietary variables were significantly associated with low MAMC and FFMI, contrasting with previous studies showing independent relationships with protein, iron and antioxidants $(9,10)$.

Insulin resistance and blood glucose were the only metabolic risk factors associated with low MAMC and FFMI. This supports research showing insulin resistance is inversely associated with low muscle mass, independent of obesity (32), and may explain why diabetics are prone to sarcopenia (33). This finding has important implications for management of insulin resistance and diabetes for sarcopenia prevention.

Two inflammatory markers (CRP and fibrinogen) were significantly associated with low MAMC and FFMI. This is consistent with other studies $(34,35)$ and a review suggesting sarcopenia is a low-level inflammatory state driven by cytokines and oxidative stress (11). These findings highlight the importance of reducing inflammation in older adults, potentially through lifestyle modifications such as increasing physical activity or an anti-inflammatory diet, especially since inflammatory markers increase with age $(22,36)$. Associations between vWF and t-PA with low muscle mass were novel and consistent with a review suggesting that endothelial dysfunction may contribute to sarcopenia development (12). Thus sarcopenia may be a pathway by which endothelial dysfunction may contribute to poorer functional status reported in other studies (37). The lack of association between D-dimer, IL-6 and homocysteine and low MAMC/FFMI supports evidence showing no consistent association between high IL-6 and sarcopenia (38), but is contrary to research showing associations between these three biomarkers and sarcopenia $(34,35)$.

The study's major strength is the use of two clinically available and easily measurable markers of muscle mass to identify lifestyle, dietary and cardiovascular risk factors. Although more precise muscle mass measures (e.g. computerized tomography, magnetic resonance imaging, dualenergy X-ray absorptiometry) were unavailable here, such expensive measures are rarely available in primary care settings and MAMC/FFMI measures represent practical alternatives. Using indirect measurements of muscle mass may have attenuated observed associations with risk factors. The lowest quartile of MAMC cut-point $(24.95 \mathrm{~cm})$ was comparable to another population-based study, which used a cut-point for the lowest tertile of $21.1 \mathrm{~cm}$ for slightly older men, aged $>80$ years (39). Similarly, the lowest quartile of FFMI cut-point (15.96 $\mathrm{kg} / \mathrm{m}^{2}$ ) was comparable to a study in men and women, where the lowest quartile cut-point was $14.6 \mathrm{~kg} / \mathrm{m}^{2}$ (40). The
European and International Working Groups on Sarcopenia have recommended using both low muscle mass and low muscle function (strength or performance) to diagnose sarcopenia $(4,7)$. However, muscle function measures were unavailable in this study and therefore findings are applicable to low muscle mass, but not necessarily to low muscle strength or performance.

This study has some limitations. Firstly, it was crosssectional so causality cannot be established and findings should therefore be interpreted with caution. The assessment of sarcopenia was based on low muscle mass, measured at one time point, since data on loss of muscle mass over time were unavailable. Some differences were observed in the associations of risk factors with MAMC and FFMI. This could be explained by the greater inaccuracy of BIA in assessment of FFMI in older people, predominantly due to fat-free mass hydration variability $(41,42)$. Non-participation bias is possible, as men too unwell to attend the physical examination would have been excluded, potentially eliminating men with extremely low muscle mass and underestimating observed associations. This may explain why some variables previously associated with low muscle mass, e.g. protein intake, were not related in this study. However, the response rate was high and BMI did not differ between respondents and non-respondents (14), suggesting the extent of this bias is limited. Since this study was in older men, predominantly of white European ethnic origin, generalisation of findings to women and nonwhite ethnic groups should be cautious. Several exposure variables were based on self-reports, so misclassification was possible. However, previous food frequency questionnaire validation reduces the risk of bias for dietary variables (19-21).

Physical inactivity, low percent energy from carbohydrates, high levels of insulin resistance, inflammation (CRP, fibrinogen) and endothelial dysfunction (vWF) are associated with low muscle mass, as measured by MAMC and FFMI, in older men. Our findings suggest that increasing physical activity, consuming a diet with a high proportion of energy from carbohydrates, and taking steps to prevent insulin resistance and reduce inflammation and endothelial dysfunction may help to reduce the risk of low muscle mass in older men. However, further longitudinal studies are needed to confirm the causality of these associations.

Acknowledgements: The British Regional Heart Study is a British Heart Foundation Research Group and also received funding from the Department of Health, England. JLA is funded by a PhD studentship by the National Institute of Health Research School for Primary Care Research.

Conflict of interest: The authors do not have any conflict of interest to disclose. 


\section{Supplementary Table 1}

Morbidity Prevalence of Participants by MAMC Quartiles

\begin{tabular}{|c|c|c|c|c|c|c|}
\hline & \multicolumn{4}{|c|}{ MAMC Quartiles (cm) } & \multirow[b]{2}{*}{$\begin{array}{l}\text { p-value for trend } \\
\text { (adjusted for age) }\end{array}$} & \multirow[b]{2}{*}{$\begin{array}{l}\text { p-value for trenc } \\
\text { (adjusted for } \\
\text { age and BMI) }\end{array}$} \\
\hline & $\leq \mathbf{2 4 . 9 5}$ & 24.95-26.45 & 26.45-27.97 & $\geq 27.97$ & & \\
\hline \multicolumn{7}{|l|}{ Respiratory } \\
\hline $\mathrm{FEV}_{1}(\mathrm{~L})$ & $2.4 \pm 0.7$ & $2.6 \pm 0.7$ & $2.6 \pm 0.6$ & $2.7 \pm 0.6$ & $<0.001$ & $<0.001$ \\
\hline \multicolumn{7}{|l|}{ Morbidity } \\
\hline CVD $(\%)$ & 24.7 & 22.4 & 22.5 & 21.2 & 0.98 & $<0.001$ \\
\hline $\mathrm{CHD}(\%)$ & 20.7 & 18.5 & 19.3 & 18.2 & 0.91 & $<0.001$ \\
\hline Stroke $(\%)$ & 6.9 & 5.7 & 5.3 & 5.6 & 0.28 & 0.24 \\
\hline Diabetes $(\%)$ & 5.9 & 6.7 & 6.7 & 7.8 & 0.01 & 0.10 \\
\hline Heart failure (\%) & 1.8 & 2.2 & 1.9 & 1.3 & 0.71 & 0.003 \\
\hline Cancer $(\%)$ & 7.5 & 6.6 & 5.1 & 5.2 & 0.19 & 0.05 \\
\hline Poor/fair self-rated health $(\%)$ & 30.7 & 25.5 & 22.4 & 25.2 & 0.007 & $<0.001$ \\
\hline \multicolumn{7}{|l|}{ Disability } \\
\hline Long-standing illness/disability (\%) & 36.2 & 33.9 & 31.5 & 34.0 & 0.26 & $<0.001$ \\
\hline Mobility limitation (\%) & 23.9 & 18.6 & 20.1 & 21.3 & 0.68 & $<0.001$ \\
\hline
\end{tabular}

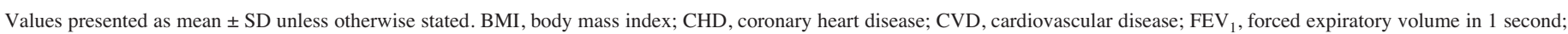
MAMC, midarm muscle circumference.

Supplementary Table 2

Morbidity Prevalence of Participants by FFMI Quartiles

\begin{tabular}{|c|c|c|c|c|c|c|}
\hline & \multicolumn{4}{|c|}{ FFMI Quartiles $\left(\mathrm{kg} / \mathrm{m}^{2}\right)$} & \multirow{2}{*}{$\begin{array}{l}\text { p value for trend } \\
\text { (adjusted for age) }\end{array}$} & \multirow{2}{*}{$\begin{array}{l}\text { p value for trend } \\
\text { (adjusted for age } \\
\text { and BMI) }\end{array}$} \\
\hline & $\leq 15.96$ & 15.96-17.09 & $17.09-18.36$ & $\geq 18.36$ & & \\
\hline \multicolumn{7}{|l|}{ Respiratory } \\
\hline $\mathrm{FEV}_{1}(\mathrm{~L})$ & $2.5 \pm 0.7$ & $2.5 \pm 0.6$ & $2.6 \pm 0.6$ & $2.6 \pm 0.6$ & $<0.001$ & $<0.001$ \\
\hline \multicolumn{7}{|l|}{ Morbidity } \\
\hline CVD $(\%)$ & 22.7 & 22.6 & 21.7 & 22.8 & 0.92 & $<0.001$ \\
\hline $\mathrm{CHD}(\%)$ & 18.7 & 19.1 & 19.1 & 19.1 & 0.78 & 0.004 \\
\hline Stroke $(\%)$ & 6.1 & 5.6 & 4.8 & 6.5 & 0.54 & 0.19 \\
\hline Diabetes (\%) & 5.3 & 5.1 & 7.4 & 9.8 & $<0.001$ & 0.08 \\
\hline Heart failure (\%) & 2.6 & 1.3 & 1.2 & 1.9 & 0.48 & 0.005 \\
\hline Cancer $(\%)$ & 7.4 & 5.7 & 5.9 & 5.5 & 0.30 & 0.18 \\
\hline Poor/fair self-rated health $(\%)$ & 29.0 & 23.8 & 23.5 & 27.6 & 0.90 & $<0.001$ \\
\hline \multicolumn{7}{|l|}{ Disability } \\
\hline Long-standing illness/disability (\%) & 33.0 & 33.5 & 31.6 & 36.4 & 0.12 & 0.10 \\
\hline Mobility limitation (\%) & 21.0 & 19.9 & 19.3 & 23.2 & 0.09 & $<0.001$ \\
\hline
\end{tabular}

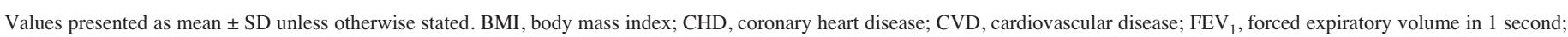
FFMI, fat free mass index.

\section{References}

1. Baumgartner RN, Koehler KM, Gallagher D, Romero L, Heymsfield SB, Ross RR, Garry PJ, Lindeman RD. Epidemiology of sarcopenia among the elderly in New Mexico. Am J Epidemiol 1998; 147: 755-763.

2. Janssen I, Heymsfield SB, Ross R. Low relative skeletal muscle mass (sarcopenia) in older persons is associated with functional impairment and physical disability. $\mathrm{J}$ Am Geriatr Soc 2002; 50: 889-896.

3. Wannamethee SG, Shaper AG, Lennon L, Whincup PH. Decreased muscle mass and increased central adiposity are independently related to mortality in older men. Am J Clin Nutr 2007; 86: 1339-1346.

4. Fielding RA, Vellas B, Evans WJ, Bhasin S, Morley JE, Newman AB, Abellan van Kan G, Andrieu S, Bauer J, Breuille D, Cederholm T, Chandler J, De Meynard C, Donini L, Harris T, Kannt A, Keime Guibert F, Onder G, Papanicolaou D, Rolland Y, Rooks D, Sieber C, Souhami E, Verlaan S, Zamboni M. Sarcopenia: an undiagnosed condition in older adults. Current consensus definition: prevalence, etiology, and consequences. International working group on sarcopenia. J Am Med Dir Assoc 2011; 12: 249-256.

5. Janssen I, Shepard DS, Katzmarzyk PT, Roubenoff R. The healthcare costs of sarcopenia in the United States. J Am Geriatr Soc 2004; 52: 80-85.

6. Morley JE. Sarcopenia: diagnosis and treatment. J Nutr Health Aging 2008; 12: 452 456.

7. Cruz-Jentoft AJ, Baeyens JP, Bauer JM, Boirie Y, Cederholm T, Landi F, Martin FC, Michel JP, Rolland Y, Schneider SM, Topinkova E, Vandewoude M, Zamboni MSDR. Sarcopenia: European consensus on definition and diagnosis: Report of the European Working Group on Sarcopenia in Older People. Age Ageing 2010; 39: 412-423.

8. Waters DL, Baumgartner RN. Sarcopenia and obesity. Clin Geriatr Med 2011; 27: 401-421.

9. Houston DK, Nicklas BJ, Ding J, Harris TB, Tylavsky FA, Newman AB, Lee JS, Sahyoun NR, Visser M, Kritchevsky SB. Dietary protein intake is associated with 
lean mass change in older, community-dwelling adults: the Health, Aging, and Body Composition (Health ABC) Study. Am J Clin Nutr 2008; 87: 150-155.

10. Volkert D. The role of nutrition in the prevention of sarcopenia. Wien Med Wochenschr 2011; 161: 409-415.

11. Jensen GL. Inflammation: roles in aging and sarcopenia. JPEN J Parenter Enteral Nutr 2008; 32: 656-659.

12. Timmerman KL, Volpi E. Endothelial function and the regulation of muscle protein anabolism in older adults. Nutr Metab Cardiovasc Dis 2012; doi: 10.1016/j.numecd.2012.03.013.

13. Shaper AG, Pocock SJ, Walker M, Cohen NM, Wale CJ, Thomson AG. British Regional Heart Study: cardiovascular risk factors in middle-aged men in 24 towns. Br Med J (Clin Res Ed) 1981; 283: 179-186.

14. Thomas MC, Walker M, Lennon LT, Thomson AG, Lampe FC, Shaper AG, Whincup PH. Non-attendance at re-examination 20 years after screening in the British Regional Heart Study. J Public Health Med 2002; 24: 285-291.

15. Heymsfield SB, McManus C, Smith J, Stevens V, Nixon DW. Anthropometric measurement of muscle mass: revised equations for calculating bone-free arm muscle area. Am J Clin Nutr 1982; 36: 680-690.

16. Deurenberg P, van der Kooij K, Evers P, Hulshof T. Assessment of body composition by bioelectrical impedance in a population aged greater than $60 \mathrm{y}$. Am J Clin Nutr 1990; 51: 3-6.

17. Ramsay SE, Whincup PH, Shaper AG, Wannamethee SG. The relations of body composition and adiposity measures to ill health and physical disability in elderly men. Am J Epidemiol 2006; 164: 459-469.

18. Noori N, Kopple JD, Kovesdy CP, Feroze U, Sim JJ, Murali SB, Luna A, Gomez M, Luna C, Bross R, Nissenson AR, Kalantar-Zadeh K. Mid-Arm Muscle Circumference and Quality of Life and Survival in Maintenance Hemodialysis Patients. Clin J Am Soc Nephrol 2010; 5: 2258-2268.

19. Bolton-Smith C, Casey CE, Gey KF, Smith WC, Tunstall-Pedoe H. Antioxidant vitamin intakes assessed using a food-frequency questionnaire: correlation with biochemical status in smokers and non-smokers. Br J Nutr 1991; 65: 337-346.

20. Yarnell JW, Fehily AM, Milbank JE, Sweetnam PM, Walker CL. A short dietary questionnaire for use in an epidemiological survey: comparison with weighed dietary records. Hum Nutr Appl Nutr 1983; 37: 103-112.

21. Bolton-Smith C, Milne AC. Food frequency v weighed intake data in Scottish men. Proc Nutr Soc 1991; 50: 36A (abstr).

22. Wannamethee SG, Lowe GD, Rumley A, Bruckdorfer KR, Whincup PH. Associations of vitamin $\mathrm{C}$ status, fruit and vegetable intakes, and markers of inflammation and hemostasis. Am J Clin Nutr 2006; 83: 567-574.

23. Willett WC, Howe GR, Kushi LH. Adjustment for total energy intake in epidemiologic studies. Am J Clin Nutr 1997; 65: 1220S-1228S.

24. Wannamethee SG, Shaper AG, Alberti KG. Physical activity, metabolic factors, and the incidence of coronary heart disease and type 2 diabetes. Arch Intern Med 2000; 160: 2108-2116.

25. Wannamethee SG, Shaper AG, Morris RW, Whincup PH. Measures of adiposity in the identification of metabolic abnormalities in elderly men. Am J Clin Nutr 2005; 81: 1313-1321.

26. Executive Summary of The Third Report of The National Cholesterol Education Program (NCEP) Expert Panel on Detection, Evaluation, And Treatment of High Blood Cholesterol In Adults (Adult Treatment Panel III). JAMA 2001; 285: 24862497.

27. Wannamethee SG, Whincup PH, Rumley A, Lowe GD. Inter-relationships of interleukin-6, cardiovascular risk factors and the metabolic syndrome among older men. J Thromb Haemost 2007; 5: 1637-1643.

28. Whincup PH, Refsum H, Perry IJ, Morris R, Walker M, Lennon L, Thomson A, Ueland PM, Ebrahim SB. Serum total homocysteine and coronary heart disease: prospective study in middle aged men. Heart 1999; 82: 448-454.

29. World Health Organization. Obesity: preventing and managing the global epidemic. Report of a WHO Consultation. Geneva, WHO 2000; WHO Technical Report Series, No 894.

30. Szulc P, Duboeuf F, Marchand F, Delmas PD. Hormonal and lifestyle determinants of appendicular skeletal muscle mass in men: the MINOS study. Am J Clin Nutr 2004; 80: 496-503.

31. World Health Organization. Diet, nutrition and the prevention of chronic disease. Report of a Joint WHO/FAO Expert Consultation. Geneva, WHO 2003; WHO Technical Report Series, No 916.

32. Srikanthan P, Hevener AL, Karlamangla AS. Sarcopenia Exacerbates ObesityAssociated Insulin Resistance and Dysglycemia: Findings from the National Health and Nutrition Examination Survey III. PLoS One 2010; 5: e10805.

33. Park SW, Goodpaster BH, Lee JS, Kuller LH, Boudreau R, de Rekeneire N, Harris TB, Kritchevsky S, Tylavsky FA, Nevitt M, Cho YW, Newman AB. Excessive loss of skeletal muscle mass in older adults with type 2 diabetes. Diabetes Care 2009; 32: 1993-1997.

34. McDermott MM, Ferrucci L, Guralnik JM, Tian L, Green D, Liu K, Tan J, Liao Y, Pearce WH, Schneider JR, Ridker P, Rifai N, Hoff F, Criqui MH. Elevated levels of inflammation, d-dimer, and homocysteine are associated with adverse calf muscle characteristics and reduced calf strength in peripheral arterial disease. J Am Coll Cardiol 2007; 50: 897-905.

35. Cesari M, Kritchevsky SB, Baumgartner RN, Atkinson HH, Penninx BW, Lenchik L, Palla SL, Ambrosius WT, Tracy RP, Pahor MSOICI-R. Sarcopenia, obesity, and inflammation--results from the Trial of Angiotensin Converting Enzyme Inhibition and Novel Cardiovascular Risk Factors study. Am J Clin Nutr 2005; 82: 428-434.

36. Hamer M, Sabia S, Batty GD, Shipley MJ, Tabak AG, Singh-Manoux A, Kivimaki M. Physical activity and inflammatory markers over 10 years: follow-up in men and women from the Whitehall II cohort study. Circulation 2012; 126: 928-933.

37. Huffman KM, Pieper CF, Kraus VB, Kraus WE, Fillenbaum GG, Cohen HJ Relations of a Marker of Endothelial Activation (s-VCAM) to Function and Mortality in Community-Dwelling Older Adults. J Gerontol A Biol Sci Med Sci 2011; 66A: 1369-1375.

38. Schaap LA, Pluijm SM, Deeg DJ, Visser M. Inflammatory markers and loss of muscle mass (sarcopenia) and strength. Am J Med 2006; 119: 526.e529-517.

39. Landi F, Russo A, Liperoti R, Pahor M, Tosato M, Capoluongo E, Bernabei R, Onder G. Midarm muscle circumference, physical performance and mortality: results from the aging and longevity study in the Sirente geographic area (ilSIRENTE study). Clin Nutr 2010; 29: 441-447.

40. Han SS, Kim KW, Kim KI, Na KY, Chae DW, Kim S, Chin HJ. Lean mass index: a better predictor of mortality than body mass index in elderly Asians. J Am Geriatr Soc 2010; 58: 312-317.

41. Roubenoff R, Baumgartner RN, Harris TB, Dallal GE, Hannan MT, Economos CD, Stauber PM, Wilson PW, Kiel DP. Application of bioelectrical impedance analysis to elderly populations. J Gerontol A Biol Sci Med Sci 1997; 52: M129-136.

42. Bussolotto M, Ceccon A, Sergi G, Giantin V, Beninca P, Enzi G. Assessment of body composition in elderly: accuracy of bioelectrical impedance analysis. Gerontology 1999; 45: 39-43. 